\title{
BIRKHOFF-KELLOGG THEOREMS ON INVARIANT DIRECTIONS FOR MULTIMAPS
}

\author{
RAVI P. AGARWAL AND DONAL O'REGAN
}

Received 11 December 2001

We establish Birkhoff-Kellogg type theorems on invariant directions for a general class of maps. Our results, in particular, apply to Kakutani, acyclic, O’Neill, approximable, admissible, and $\boldsymbol{U}_{c}^{\kappa}$ maps.

\section{Introduction}

This paper presents Birkhoff-Kellogg type theorems on invariant directions for a large class of maps. A number of results which will enable to deduce results for upper semicontinuous maps which are either (a) Kakutani, (b) acyclic, (c) O’Neill, or (d) admissible (strongly) in the sense of Gorniewicz are given.

The results in this paper, when the map is compact, complement and extend the previously known results in $[8,14,16]$. Also using the results in [7], we are able to present invariant direction results for countably condensing maps.

For the remainder of this section, we present some definitions and some known facts. Let $X$ and $Y$ be subsets of Hausdorff topological vector spaces $E_{1}$ and $E_{2}$, respectively. We will look at maps $F: X \rightarrow K(Y)$, here $K(Y)$ denotes the family of nonempty compact subsets of $Y$. We say $F: X \rightarrow K(Y)$ is Kakutani if $F$ is upper semicontinuous with convex values. A nonempty topological space is said to be acyclic, if all its reduced C̆ech homology groups over the rationals are trivial. Now $F: X \rightarrow K(Y)$ is acyclic if $F$ is upper semicontinuous with acyclic values. The map $F: X \rightarrow K(Y)$ is said to be an O'Neill map if $F$ is continuous and if the values of $F$ consist of one or $m$-acyclic components (here $m$ is fixed).

Given two open neighborhoods $U$ and $V$ of the origins in $E_{1}$ and $E_{2}$, respectively, a $(U, V)$-approximate continuous selection [6] of $F: X \rightarrow K(Y)$ is a continuous function $s: X \rightarrow Y$ satisfying

$$
s(x) \in(F[(x+U) \cap X]+V) \cap Y, \quad \forall x \in X .
$$

Copyright (C) 2003 Hindawi Publishing Corporation

Abstract and Applied Analysis 2003:7 (2003) 435-448

2000 Mathematics Subject Classification: $47 \mathrm{H} 10$

URL: http://dx.doi.org/10.1155/S1085337503205030 
We say $F: X \rightarrow K(Y)$ is approximable if its restriction $\left.F\right|_{K}$, to any compact subset $K$ of $X$, admits a $(U, V)$-approximate continuous selection for every open neighborhood $U$ and $V$ of the origins in $E_{1}$ and $E_{2}$, respectively.

For our next definition, let $X$ and $Y$ be metric spaces. A continuous singlevalued map $p: Y \rightarrow X$ is called a Vietoris map if the following two conditions are satisfied:

(i) for each $x \in X$, the set $p^{-1}(x)$ is acyclic;

(ii) $p$ is a proper map, that is, for every compact $A \subseteq X, p^{-1}(A)$ is compact.

Definition 1.1. A multifunction $\phi: X \rightarrow K(Y)$ is admissible (strongly) in the sense of Gorniewicz if $\phi: X \rightarrow K(Y)$ is upper semicontinuous, and if there exist a metric space $Z$ and two continuous maps $p: Z \rightarrow X$ and $q: Z \rightarrow Y$ such that

(i) $p$ is a Vietoris map;

(ii) $\phi(x)=q\left(p^{-1}(x)\right)$ for any $x \in X$.

Remark 1.2. It should be noted [10, page 179] that $\phi$ upper semicontinuous is redundant in Definition 1.1.

Suppose $X$ and $Y$ are Hausdorff topological spaces. Given a class $\mathscr{X}$ of maps, $\mathscr{L}(X, Y)$ denotes the set of maps $F: X \rightarrow 2^{Y}$ (nonempty subsets of $Y$ ) belonging to $\mathscr{X}$, and $\mathscr{X}_{c}$ the set of finite compositions of maps in $\mathscr{X}$. A class $\mathscr{U}$ of maps is defined by the following properties:

(i) $\mathcal{U}$ contains the class $\mathscr{C}$ of single-valued continuous functions;

(ii) each $F \in U_{c}$ is upper semicontinuous and compact valued;

(iii) for any polytope $P, F \in U_{c}(P, P)$ has a fixed-point where the intermediate spaces of composites are suitably chosen for each $\boldsymbol{U}$.

Definition 1.3. The map $F \in \mathcal{U}_{c}^{\kappa}(X, Y)$ if for any compact subset $K$ of $X$, there is a $G \in U_{c}(K, Y)$ with $G(x) \subseteq F(x)$ for each $x \in K$.

Examples of $\boldsymbol{u}_{c}^{\kappa}$ maps are the Kakutani, the acyclic, the O'Neill maps, and the maps admissible in the sense of Gorniewicz.

For a subset $K$ of a topological space $X$, we denote by $\operatorname{Cov}_{X}(K)$ the directed set of all coverings of $K$ by open sets of $X$ (usually we write $\operatorname{Cov}(K)=\operatorname{Cov}_{X}(K)$ ). Given two maps $F, G: X \rightarrow 2^{Y}$ and $\alpha \in \operatorname{Cov}(Y), F$ and $G$ are said to be $\alpha$-close, if for any $x \in X$, there exists $U_{x} \in \alpha, y \in F(x) \cap U_{x}$, and $w \in G(x) \cap U_{x}$.

By a space, we mean a Hausdorff topological space. In what follows, $Q$ denotes a class of topological spaces. A space $Y$ is an extension space for $Q$ (written $Y \in \mathrm{ES}(Q))$ if for any pair $(X, K)$ in $Q$ with $K \subseteq X$ closed, any continuous function $f_{0}: K \rightarrow Y$ extends to a continuous function $f: X \rightarrow Y$.

A space $Y$ is an approximate extension space for $Q$ (and we write $Y \in \operatorname{AES}(Q)$ ) if for any $\alpha \in \operatorname{Cov}(Y)$ and any pair $(X, K)$ in $Q$ with $K \subseteq X$ closed and any continuous function $f_{0}: K \rightarrow Y$, there exists a continuous function $f: X \rightarrow Y$ such that $\left.f\right|_{K}$ is $\alpha$-close to $f_{0}$. 
Definition 1.4. Let $V$ be a subset of a Hausdorff topological vector space $E$. Then we say $V$ is Schauder admissible if for every compact subset $K$ of $V$ and every covering $\alpha \in \operatorname{Cov}_{V}(K)$, there exists a continuous function (called the Schauder projection) $\pi_{\alpha}: K \rightarrow V$ such that

(i) $\pi_{\alpha}$ and $i: K \rightarrow V$ are $\alpha$-close;

(ii) $\pi_{\alpha}(K)$ is contained in a subset $C \subseteq V$ with $C \in$ AES (compact).

If $V \in$ AES (compact), then $V$ is trivially Schauder admissible. If $V$ is an open convex subset of a Hausdorff locally convex topological space $E$, then it is well known that $V$ is Schauder admissible.

The following fixed-point result was established in [5].

TheOREM 1.5. Let $V$ be a Schauder admissible subset of a Hausdorff topological vector space $E$ and $F \in U_{c}^{\kappa}(V, V)$ a compact map. Then $F$ has a fixed point.

A nonempty subset $X$ of a Hausdorff topological vector space $E$ is said to be admissible if for every compact subset $K$ of $X$ and every neighborhood $V$ of 0 , there exists a continuous map $h: K \rightarrow X$ with $x-h(x) \in V$ for all $x \in K$ and $h(K)$ is contained in a finite-dimensional subspace of $E$. The nonempty subset $X$ is said to be $q$-admissible if any nonempty compact, convex subset $\Omega$ of $X$ is admissible.

In [12], we proved the following fixed-point result.

THeorem 1.6. Let $\Omega$ be a q-admissible, closed, convex subset of a Hausdorff topological vector space $E$ with $x_{0} \in \Omega$. Suppose $F \in U_{c}^{\kappa}(\Omega, \Omega)$ with the following property holding:

$$
A \subseteq \Omega, A=\overline{\mathrm{co}}\left(\left\{x_{0}\right\} \cup F(A)\right) \text {, implies } A \text { is compact. }
$$

Then $F$ has a fixed point in $\Omega$.

Let $(E, d)$ be a pseudometric space. For $S \subseteq E$, let $B(S, \epsilon)=\{x \in E: d(x, S) \leq$ $\epsilon\}, \epsilon>0$, where $d(x, S)=\inf _{y \in Y} d(x, y)$. The measure of noncompactness of the set $M \subseteq E$ is defined by $\alpha(M)=\inf Q(M)$ where

$$
Q(M)=\{\epsilon>0: M \subseteq B(A, \epsilon) \text { for some finite subset } A \text { of } E\} .
$$

Let $E$ be a locally convex Hausdorff topological vector space and let $P$ be a defining system of seminorms on $E$. Suppose $F: S \rightarrow 2^{E}$, here $S \subseteq E$. The map $F$ is said to be a countably $P$-concentrative mapping if $F(S)$ is bounded, and for $p \in P$, for each countably bounded subset $X$ of $S$, we have $\alpha_{p}(F(X)) \leq \alpha_{p}(X)$, and for $p \in P$, for each countably bounded non- $p$-precompact subset $X$ of $S$ (i.e., $X$ is not precompact in the pseudonormed space $(E, p))$, we have $\alpha_{p}(F(X))<\alpha_{p}(X)$, here $\alpha_{p}(\cdot)$ denotes the measure of noncompactness in the pseudonormed space $(E, p)$.

Finally for completeness, we also give the definition of countably $k$-set contractive maps. Let $X$ be a metric space and $P_{B}(X)$ the bounded subsets of $X$. 
The Kuratowskii measure of noncompactness is the map $\alpha: P_{B}(X) \rightarrow[0, \infty)$ defined by

$$
\alpha(A)=\inf \left\{\epsilon>0: A \subseteq \cup_{i=1}^{n} X_{i}, \operatorname{diam}\left(X_{i}\right) \leq \epsilon\right\}
$$

here $A \in P_{B}(X)$. Let $S$ be a nonempty subset of $X$ and $H: S \rightarrow 2^{X}$. The map $H$ is called countably $k$-set contractive $(k \geq 0)$ if $H(S)$ is bounded and $\alpha(H(\Omega)) \leq$ $k \alpha(\Omega)$ for all countably bounded sets $\Omega$ of $S$.

\section{Hausdorff locally convex topological vector spaces}

In this section, we present a variety of Birkhoff-Kellogg type theorems on invariant directions. Throughout, $E$ will be a Hausdorff locally convex topological vector space, $C$ will be a closed convex subset of $E, U \subseteq C$ will be convex, $U$ will be an open subset of $E$, and $0 \in U$. Notice int $_{C} U=U$ since $U$ is open in $C$. Also we wish to consider maps $F: \bar{U} \rightarrow K(C)$ which are upper semicontinuous and either (a) approximable, (b) admissible (strongly) in the sense of Gorniewicz, or more generally (c) $U_{c}^{\kappa}$, here $\bar{U}$ denotes the closure of $U$ in $C$ and $K(C)$ denotes the family of nonempty compact subsets of $C$.

To take care of all the above maps (and even more general types), we introduce the following definition.

Definition 2.1. The map $F \in \operatorname{LS}(\bar{U}, C)$ if $F: \bar{U} \rightarrow K(C)$ is upper semicontinuous and satisfies condition $(D)$. We assume condition $(D)$ is

for any map $F \in L S(\bar{U}, C)$ and any continuous single-valued map $r: E \rightarrow \bar{U}, \quad r F$ satisfies condition $(D)$.

Certainly if condition ( $D$ ) means (a), (b), or (c) above, then (2.1) holds (see $[2,6,10,15])$.

Throughout this section, we will assume the map $F: \bar{U} \rightarrow K(C)$ satisfies one of the following conditions:

(H1) $F$ is compact;

(H2) if $D \subseteq \bar{U}$ and $D \subseteq \overline{\mathrm{CO}}(\{0\} \cup F(D))$, then $\bar{D}$ is compact; or

(H3) $F$ is countably $P$-concentrative and $E$ is Fréchet (here $P$ is a defining system of seminorms).

Fix $i \in\{1,2,3\}$.

Definition 2.2. We say $F \in L S^{i}(\bar{U}, C)$ if $F \in L S(\bar{U}, C)$ satisfies $(\mathrm{Hi})$.

Remark 2.3. Throughout this section, it is possible to replace $F$ upper semicontinuous in Definition 2.1 with $F$ closed and taking compact sets into relatively compact sets.

The following result was established in [4]. 
Theorem 2.4. Fix $i \in\{1,2,3\}$ and let $E$ be a Hausdorff locally convex topological vector space, $C$ a closed convex subset of $E, U \subseteq C$ convex, $U$ an open subset of $E$, $0 \in U$, and assume (2.1) holds. Suppose $F \in L S^{i}(\bar{U}, C)$ and assume the following condition holds:

$$
\text { any map } \Phi \in L S^{i}(\bar{U}, \bar{U}) \text { has a fixed point. }
$$

Then either

(i) F has a fixed point in $\bar{U}$; or

(ii) there exist $x \in \partial U$ and $\lambda \in(0,1)$ with $x \in \lambda F x$;

here $\partial U$ denotes the boundary of $U$ in $C$.

Example 2.5. Suppose condition $(D)$ in Definition 2.1 means $F: \bar{U} \rightarrow K(C)$ belongs to $U_{c}^{\kappa}(\bar{U}, C)$. Now since $U_{c}^{\kappa}$ is closed under compositions, then (2.1) is true. If $i=1$, we know from [15] that (2.2) holds. If $i=2$, we know from [13] that (2.2) is satisfied. If $i=3$, we know from [11] that (2.2) holds. As a result, Theorem 2.4 contains most of the Leray-Schauder alternatives (see $[4,14,16,17]$ and the references therein).

For our next result, assume condition $(D)$ is such that

$$
\text { for any map } F \in L S(\bar{U}, C) \text { and any } \lambda \in \mathbb{R}, \lambda F \text { satisfies condition }(D) \text {. }
$$

Certainly if condition (D) means (a) or (b) above, then (2.3) is satisfied.

Now from Theorem 2.4, we obtain the following Birkhoff-Kellogg type theorem. Some of the ideas here were borrowed from the literature (see [14] and the references therein).

Theorem 2.6. Let E be a Hausdorff locally convex topological vector space, $C$ a closed convex subset of $E, U \subseteq C$ convex, $U$ an open subset of $E, 0 \in U$, and assume (2.1), (2.2) (with $i=1$ ), and (2.3) hold. Suppose $F \in L S^{1}(\bar{U}, C)$ and assume the following condition holds:

$$
\exists \mu \in \mathbb{R}, \quad \text { with } \mu F(\bar{U}) \cap \bar{U}=\varnothing .
$$

Then there exist $\lambda \in(0,1)$ and $x \in \partial U$ with $\left(\lambda^{-1} \mu^{-1}\right) x \in F x$ (i.e., $\left.F\right|_{\partial U}$ has an eigenvalue); here $\mu \neq 0$ is chosen as in (2.4).

Remark 2.7. Notice that $0 \in U$ guarantees that $\mu \neq 0$ in (2.4).

Proof. Let $\mu \neq 0$ be chosen as in (2.4). Now (2.3) guarantees that $\mu F \in L S(\bar{U}, C)$, and as a result $\mu F \in L S^{1}(\bar{U}, C)$. In addition, (2.4) guarantees that $\mu F$ has no fixed points in $\bar{U}$. Theorem 2.4 (applied to $\mu F$ ) guarantees that there exists $\lambda$ and $x \in$ $\partial U$ with $x \in \lambda(\mu F) x$. As a result, $\left(\lambda^{-1} \mu^{-1}\right) x \in F x$ and the proof is complete.

Example 2.8. In Theorem 2.6, if condition $(D)$ means that the map $F: \bar{U} \rightarrow K(C)$ is either (a) approximable, or (b) admissible in the sense of Gorniewicz, then we know that (2.1), (2.2) (see [3, 12, 13]), and (2.3) hold. 
In Theorem 2.6, if condition $(D)$ means that the map $F: \bar{U} \rightarrow K(C)$ belongs to $u_{c}^{\kappa}(\bar{U}, C)$, then we know that (2.1) and (2.2) hold. Notice that (2.3) may not be true. However, (2.3) (or a slight modification of it, see (2.5)) may work for a subclass $\mathscr{A}(\bar{U}, C)$ of $U_{c}^{\kappa}(\bar{U}, C)$ (e.g., $\mathscr{A}$ could be the Kakutani or acyclic maps or indeed the maps described in the above example). In the proof of our next result, condition $(D)$ means that the map $F: \bar{U} \rightarrow K(C)$ belongs to $u_{c}^{\kappa}(\bar{U}, C)$, so $F \in L S(\bar{U}, C)$ means that $F$ is upper semicontinuous and $F \in U_{c}^{\kappa}(\bar{U}, C)$.

Theorem 2.9. Let $E$ be a Hausdorff locally convex topological vector space, $C$ a closed convex subset of $E, U \subseteq C$ convex, $U$ an open subset of $E, 0 \in U, F \in$ $\mathcal{A}(\bar{U}, C)$ a compact map, and assume (2.4) holds. Suppose the following condition holds:

$$
\text { for any map } F \in \mathscr{A}(\bar{U}, C) \text {, and any } \lambda \in \mathbb{R}, \lambda F \in \mathcal{U}_{c}^{\kappa}(\bar{U}, C) \text {. }
$$

Then there exist $\lambda \in(0,1)$ and $x \in \partial U$ with $\left(\lambda^{-1} \mu^{-1}\right) x \in F x$ (i.e., $\left.F\right|_{\partial U}$ has an eigenvalue); here $\mu \neq 0$ is chosen as in (2.4).

Proof. Essentially the same reasoning as in Theorem 2.6 establishes the result.

In our next result, we assume (2.3) when $|\lambda| \leq 1$.

Theorem 2.10. Fix $i \in\{2,3\}$ and let $E$ be a Hausdorff locally convex topological vector space, $C$ a closed convex subset of $E, U \subseteq C$ convex, $U$ an open subset of $E$, $0 \in U, F \in L S^{i}(\bar{U}, C)$, and assume (2.1) and (2.2) hold. In addition, suppose the following conditions are satisfied:

(i) for any map $F \in L S(\bar{U}, C)$ and any $\lambda \in \mathbb{R}$ with $|\lambda| \leq 1, \lambda F$ satisfies condition (D),

(ii) there exists $\mu \in \mathbb{R}$ with $|\mu| \leq 1, \mu F(\bar{U}) \cap \bar{U}=\varnothing$,

(iii) if $i=2$, assume either $\mu>0$ in (ii) or $-F(D)=F(D)$ for any $D \subseteq \bar{U}$.

Then there exists $\lambda \in(0,1)$ and $x \in \partial U$ with $\left(\lambda^{-1} \mu^{-1}\right) x \in F x$.

Proof. Let $\mu \neq 0$ be chosen as in (i), and notice that $\mu F \in L S(\bar{U}, C)$ from (i). We claim

$$
\mu F \in L S^{i}(\bar{U}, C)
$$

If $i=3$, then (2.6) is immediate since $|\mu| \leq 1$. Next suppose $i=2$ and let $D \subseteq$ $\bar{U}$ with $D \subseteq \overline{\mathrm{co}}(\{0\} \cup \mu F(D))$. Now from Theorem 2.10(iii), we have $\mu F(D) \subseteq$ $\operatorname{co}(\{0\} \cup F(D))$, and so

$$
D \subseteq \overline{\mathrm{CO}}(\{0\} \cup \operatorname{co}(\{0\} \cup F(D)))=\overline{\mathrm{co}}(\operatorname{co}(\{0\} \cup F(D)))=\overline{\mathrm{CO}}(\{0\} \cup F(D)) .
$$

Now $\bar{D}$ is compact since $F \in L S^{2}(\bar{U}, C)$, and so (2.6) holds if $i=2$. Apply Theorem 2.4 to $\mu F$. 
Remark 2.11. In Theorem 2.10, condition (iii) can be replaced by the following more general condition:

$$
\begin{aligned}
& \text { if } i=2 \text { and if } D \subseteq \bar{U} \text { with } D \subseteq \overline{\mathrm{co}}(\{0\} \cup \mu F(D)), \\
& \text { then } \bar{D} \text { is compact, here } \mu \text { is chosen as in (ii) }
\end{aligned}
$$

(of course with this assumption, we do not need to assume that $|\mu| \leq 1$ in (ii) if $i=2$ ). For example, if $F$ is $P$-concentrative (here $E$ is Fréchet), then clearly (2.8) is satisfied (if $|\mu| \leq 1$ ).

Remark 2.12. It is also possible to use Theorem 2.9 to obtain an analogue of Theorem 2.10 for the subclass $\mathcal{A}$. We leave the details to the reader.

In Theorem 2.6 (resp., Theorem 2.10), if $\mu>0$ in (2.4) (resp., (ii)), we say that $\left.F\right|_{\partial U}$ has an invariant direction (i.e., has a positive eigenvalue). Some of the ideas here were borrowed from the literature (see [14] and the references therein).

Theorem 2.13. Let $E=(E,\|\cdot\|)$ be an infinite-dimensional normed linear space, $C=E, U=B, F \in L S^{1}(\bar{B}, E)$, and assume that (2.1), (2.2) (with $i=1$ ), and (2.3) hold; here $B=\{x \in E:\|x\|<1\}$. In addition, suppose the following two conditions are satisfied:

$$
\begin{gathered}
\text { for any continuous map } r: \bar{B} \longrightarrow S, \text { Fr satisfies condition }(D), \\
\qquad 0 \notin \overline{F(S)}
\end{gathered}
$$

here $S=\{x \in E:\|x\|=1\}$. Then $F$ has an invariant direction.

Proof. We know [7] that there exists a continuous retraction $r: \bar{B} \rightarrow S$. Let $G=F r$ and notice that $G \in L S(\bar{B}, E)$ from (2.9). Now we claim that there exists $\mu>0$ with

$$
\mu F(S) \cap \bar{B}=\varnothing
$$

If this is true, then

$$
\mu G(\bar{B}) \cap \bar{B}=\varnothing,
$$

and so Theorem 2.6 (applied to $G$ with $U=B$ and $C=E$ ) guarantees that there exist $\lambda \in(0,1)$ and $x \in \partial B=S$ with $\lambda^{-1} \mu^{-1} x \in G x=F r x=F x$. The proof is finished. It remains to prove (2.11) but this is immediate since $0 \notin \overline{F(S)}$ (i.e., if (2.11) was false, then for each $n \in\{1,2, \ldots\}$, there exist $y_{n} \in F(S)$ and $w_{n} \in \bar{B}$ with $\left.y_{n}=(1 / n) w_{n}\right)$.

Remark 2.14. In Theorem 2.13, we can replace $B$ by any open set $U$ of $E$ with $0 \in U$ (here $E$ is any Hausdorff locally convex topological vector space) provided that $\partial U$ is a retract of $\bar{U}$, and in this case (2.10) is replaced by the following condition: $\exists \mu>0$ with $\mu F(\partial U) \cap \bar{U}=\varnothing$. 
Remark 2.15. In Theorem 2.13, $F \in L S^{1}(\bar{B}, E)$ could be replaced by $F \in L S^{1}(S, E)$. Example 2.16. In Theorem 2.13, if condition $(D)$ means that the map $F: \bar{B} \rightarrow$ $K(E)$ is either (a) approximable, or (b) admissible in the sense of Gorniewicz, then we know that (2.1), (2.2), (2.3), and (2.9) hold.

Example 2.17. In Theorem 2.13, if condition $(D)$ means that the map $F: \bar{B} \rightarrow$ $K(E)$ belongs to $u_{c}^{\kappa}(\bar{U}, C)$, then we know that (2.1), (2.2), and (2.9) are satisfied. It is possible to use Theorem 2.9 to obtain an analogue of Theorem 2.13 for the subclass $\mathscr{A}$ of $\boldsymbol{U}_{c}^{\kappa}$. We leave the details to the reader.

In [7], the authors show that if $E$ is an infinite-dimensional normed linear space, then there exists a Lipschitzian retraction $r: \bar{B} \rightarrow S$ with Lipschitz constant $k_{0}(E)$, here $B$ and $S$ are as in Theorem 2.13. In fact there exists a $k_{0}$ with $k_{0}(E) \leq$ $k_{0}$ for any space $E$ (as described above). We refer the reader to [9, Chapter 21] for a discussion of upper and lower bounds for $k_{0}(E)$, note in particular that $k_{0}(E) \geq 3$. For our next theorem, we let

$$
\begin{aligned}
& r: \bar{B} \longrightarrow S \text { be a Lipschitzian retraction } \\
& \text { with Lipschitz constant } k_{0}(E) .
\end{aligned}
$$

Theorem 2.18. Let $E=(E,\|\cdot\|)$ be an infinite-dimensional normed linear space, $C=E, U=B, F \in L S(\bar{B}, E)$, and assume that (2.1), (2.2) (with $i=3$ ), Theorem 2.10(i), (2.9), and (2.13) hold; here $B=\{x \in E:\|x\|<1\}$ and $S=\{x \in E:\|x\|=$ $1\}$. In addition, suppose the following two conditions are satisfied:

(a) $F$ is countably $k$-set contractive with $0 \leq k<1 / k_{0}(E)$, here $k_{0}(E)$ is as in (2.13);

(b) there exist $\mu>0$ with $0<\mu \leq 1, \mu F(S) \cap \bar{B}=\varnothing$.

Then $F$ has an invariant direction.

Proof. Let $G=F r$ where $r$ is as in (2.13). Notice that $G \in L S(\bar{B}, E)$ and it is easy to check that $G$ is countably $k k_{0}(E)$-set contractive. Thus, $G \in L S^{3}(\bar{B}, E)$. Now apply Theorem 2.10 to $G$.

Remark 2.19. In Theorem $2.18, F \in L S^{1}(\bar{B}, E)$ could be replaced by $F \in L S^{1}(S, E)$.

Remark 2.20. Theorem 2.18 is the first invariant direction result, to our knowledge, for countably contractive maps.

Remark 2.21. We note that the results in this section improve those in $[8,14,16]$.

\section{Hausdorff topological vector spaces}

Throughout this section, $E$ will be a Hausdorff topological vector space, $C$ a closed convex subset of $E, U$ an open subset of $C$, and $0 \in U$. This section also presents Birkhoff-Kellogg type theorems, and in some cases the results in Section 2 will be improved. 
Definition 3.1. The map $F \in G A(\bar{U}, C)$ if $F: \bar{U} \rightarrow K(C)$ is upper semicontinuous and satisfies condition $(C)$, here $\bar{U}$ denotes the closure of $U$ in $C$. We assume condition $(C)$ is

for any map $F \in G A(\bar{U}, C)$ and any continuous single-valued $\operatorname{map} \mu: \bar{U} \rightarrow[0,1], \quad \mu F$ satisfies condition $(C)$.

Certainly if condition $(C)$ means that the map $F: \bar{U} \rightarrow K(C)$ is (a) Kakutani, (b) acyclic, (c) O'Neill, (d) approximable, or (e) admissible (strongly) in the sense of Gorniewicz, then (3.1) holds.

Fix $i \in\{1,2,3\}$.

Definition 3.2. We say that $F \in G A^{i}(\bar{U}, C)$ if $F \in G A(\bar{U}, C)$ satisfies (Hi), here $(\mathrm{Hi})$ is as in Section 2.

Definition 3.3. We say that $F \in G A_{\partial U}^{i}(\bar{U}, C)$ if $F \in G A^{i}(\bar{U}, C)$ with $x \notin F(x)$ for $x \in \partial U$, here $\partial U$ denotes the boundary of $U$ in $C$.

Definition 3.4. A map $F \in G A_{\partial U}^{i}(\bar{U}, C)$ is essential in $G A_{\partial U}^{i}(\bar{U}, C)$ if for every $G \in G A_{\partial U}^{i}(\bar{U}, C)$ with $\left.G\right|_{\partial U}=\left.F\right|_{\partial U}$, there exists $x \in U$ with $x \in G(x)$.

Remark 3.5. Throughout this section, it is possible to replace $F$ upper semicontinuous in Definition 3.1 with $F$ closed and taking compact sets into relatively compact sets.

The following result was established in [4].

Theorem 3.6. Fix $i \in\{1,2,3\}$ and let $E$ be a Hausdorff topological vector space, $C$ a closed convex subset of $E, U$ an open subset of $C, 0 \in U$, and assume (3.1) holds. Suppose $F \in G A^{i}(\bar{U}, C)$ and assume the following condition is satisfied:

$$
\text { the zero map is essential in } G A_{\partial U}^{i}(\bar{U}, C) \text {. }
$$

Then either

(i) F has a fixed point in $\bar{U}$; or

(ii) there exist $x \in \partial U$ and $\lambda \in(0,1)$ with $x \in \lambda F x$.

Examples. (1) Suppose condition $(C)$ in Definition 3.1 means $F: \bar{U} \rightarrow A K(C)$, here $A K(C)$ denotes the family of nonempty, acyclic, compact subsets of $C$. Then if $i=3$ (in particular $E$ is Fréchet), we know from [3, Theorem 2.2] and [13, Theorem 2.6] that (3.2) (and of course (3.1)) is satisfied.

(2) Suppose condition $(C)$ in Definition 3.1 means that $F: \bar{U} \rightarrow K(C)$ is approximable. Then if $i=3$, we know from [3, Theorem 2.2] and [13, Theorem 2.6] that (3.2) (and of course (3.1)) holds.

(3) Suppose condition $(C)$ in Definition 3.1 means that $F: \bar{U} \rightarrow K(C)$ is admissible in the sense of Gorniewicz, $E$ is a Fréchet space ( $P$ a defining system of seminorms), $U$ is convex, and $C=E$. Now [10] guarantees that (3.1) is true. 
Now we show that (3.2) is satisfied if $i=1,2$, or 3 (in fact if $i=1$, it is enough (see Theorem 1.5) for $E$ to be a metrizable locally convex topological vector space).

To see (3.2), let $\theta \in G A_{\partial U}^{i}(\bar{U}, E)$ with $\left.\theta\right|_{\partial U}=\{0\}$. We must show that there exists $x \in U$ with $x \in \theta(x)$. Let $\mu$ be the Minkowski functional on $\bar{U}$ and let $r: E \rightarrow \bar{U}$ be given by

$$
r(x)=\frac{x}{\max \{1, \mu(x)\}}, \quad \text { for } x \in E
$$

Consider $G=r \theta$. We know [10] that $G$ is admissible in the sense of Gorniewicz, and as a result $G \in G A(\bar{U}, \bar{U})$. If $i=1$, then $G$ is compact whereas if $i=3$, then $G$ is countable $P$-concentrative since $r(A) \subseteq \operatorname{co}(A \cup\{0\})$ for any subset $A$ of $E$. Now let $i=2$ and let $D \subseteq \bar{U}$ with $D=\overline{\mathrm{co}}(\{0\} \cup G(D))$. Then since $r(A) \subseteq \operatorname{co}(A \cup$ $\{0\})$ for any subset $A$ of $E$, we have

$$
D \subseteq \overline{\operatorname{co}}(\{0\} \cup \operatorname{co}(\theta(D) \cup\{0\}))=\overline{\operatorname{co}}(\{0\} \cup \theta(D))
$$

Thus, $\bar{D}$ is compact since $\theta \in G A^{2}(\bar{U}, E)$. Now [12, Theorem 2.1] and [13, Theorem 2.2] (or alternatively Theorem 1.5, Theorem 1.6 if $i=1$ or 2 ) guarantee that there exists $x \in \bar{U}$ with $x \in G(x)=r \theta(x)$. Thus, $x=r(y)$ for some $y \in \theta x$, here $x \in \bar{U}=U \cup \partial U$ (note $C=E$ here). Suppose $x \in \partial U$. Then $\mu(x)=1$ and so

$$
1=\mu(x)=\mu(r(y))=\frac{\mu(y)}{\max \{1, \mu(y)\}}, \quad \text { since } r(y)=\frac{y}{\max \{1, \mu(y)\}} .
$$

Thus, $\mu(y) \geq 1$ and so $x=r(y)=y / \mu(y)$. This implies

$$
x \in \lambda \theta(x)=\{0\} \quad \text { since }\left.\theta\right|_{\partial U}=\{0\} ; \quad \text { here } \lambda=\frac{1}{\mu(y)} .
$$

This is a contradiction since $0 \in U$. As a result $x \in U$. This implies $\mu(x)<1$. Consequently,

$$
1>\mu(x)=\mu(r(y))=\frac{\mu(y)}{\max \{1, \mu(y)\}},
$$

and so $\mu(y)<1$. Thus $r(y)=y$, so $x=y \in \theta(x)$. As a result, (3.2) holds.

(4) Suppose condition $(C)$ in Definition 3.1 means that $F: \bar{U} \rightarrow K(C)$ is either (a) Kakutani, (b) acyclic, (c) O'Neill, or (d) approximable and $C$ is Schauder admissible. If $i=1$, then we know from [4] that (3.2) and also (3.1) hold. 
(5) Suppose condition $(C)$ in Definition 3.1 means that $F: \bar{U} \rightarrow K(C)$ is either (a) Kakutani, (b) acyclic, (c) O'Neill, or (d) approximable and $C$ is $q$-admissible with the extra condition that

$$
\overline{\mathrm{co}}(K) \text { is compact for any compact subset } K \text { of } E \text {. }
$$

If $i=2$, then we know $[4,1]$ (we use Theorem 1.6) that (3.2) and also (3.1) hold.

For our next result, assume condition $(C)$ is

for any map $F \in G A(\bar{U}, C)$ and any $\lambda \in \mathbb{R}, \quad \lambda F$ satisfies condition $(C) . \quad$ (3.9)

Theorem 3.7. Let $E$ be a Hausdorff topological vector space, $C$ a closed convex subset of $E, U$ an open subset of $C, 0 \in U$, and assume (3.1), (3.2) (with $i=1$ ), and (3.9) hold. Suppose $F \in G A^{1}(\bar{U}, C)$ and assume the following condition holds:

$$
\exists \mu \in \mathbb{R}, \quad \text { with } \mu F(\bar{U}) \cap \bar{U}=\varnothing .
$$

Then there exist $\lambda \in(0,1)$ and $x \in \partial U$ with $\left(\lambda^{-1} \mu^{-1}\right) x \in F x$, here $\mu \neq 0$ is chosen as in (3.10).

Proof. Apply Theorem 3.6 to $\mu F$ (see the proof of Theorem 2.6).

Example 3.8. In Theorem 3.7, if condition $(C)$ means the map $F: \bar{U} \rightarrow K(C)$ is either (a) Kakutani, (b) acyclic, (c) O'Neill, or (d) approximable, and $C$ is Schauder admissible, then (3.1), (3.2), and (3.9) hold.

Example 3.9. In Theorem 3.7, if condition $(C)$ means that the map $F: \bar{U} \rightarrow K(C)$ is admissible in the sense of Gorniewicz, $E$ is a Fréchet space, $U$ is convex, and $C=E$, then (3.1), (3.2), and (3.9) hold.

For our next result, we assume (3.9) when $|\lambda| \leq 1$.

Theorem 3.10. Fix $i \in\{2,3\}$ and let $E$ be a Hausdorff topological vector space, $C$ a closed convex subset of $E, U$ an open subset of $C, 0 \in U, F \in G A^{i}(\bar{U}, C)$, and assume (3.1) and (3.2) hold. In addition, suppose the following conditions are satisfied:

(a) for any map $F \in G A(\bar{U}, C)$ and any $\lambda \in \mathbb{R}$ with $|\lambda| \leq 1, \lambda F$ satisfies condition $(C)$,

(b) there exist $\mu \in \mathbb{R}$ with $|\mu| \leq 1, \mu F(\bar{U}) \cap \bar{U}=\varnothing$,

(c) if $i=2$, assume either $\mu>0$ in (b) or $-F(D)=F(D)$ for any $D \subseteq \bar{U}$.

Then there exist $\lambda \in(0,1)$ and $x \in \partial U$ with $\left(\lambda^{-1} \mu^{-1}\right) x \in F x$.

Proof. Apply Theorem 3.6 to $\mu F$ (see the proof of Theorem 2.10). 
Remark 3.11. In Theorem 3.10, (c) can be replaced by the more general condition

$$
\begin{aligned}
& \text { if } i=2 \text { and if } D \subseteq \bar{U} \text { with } D \subseteq \overline{c o}(\{0\} \cup \mu F(D)) \text {, } \\
& \text { then } \bar{D} \text { is compact, here } \mu \text { is chosen as in (b) }
\end{aligned}
$$

(of course with this assumption, we do not need to assume $|\mu| \leq 1$ in (b) if $i=2$ ).

Theorem 3.12. Let $E=(E,\|\cdot\|)$ be an infinite-dimensional normed linear space, $C=E, U=B, F \in G A^{1}(\bar{B}, E)$, and assume (3.1), (3.2) (with $i=1$ ), and (3.9) hold, here $B=\{x \in E:\|x\|<1\}$. In addition, suppose the following two conditions are satisfied:

$$
\begin{gathered}
\text { for any continuous map } r: \bar{B} \longrightarrow S, \quad \text { Fr satisfies condition }(C), \\
0 \notin \overline{F(S),}
\end{gathered}
$$

here $S=\{x \in E:\|x\|=1\}$. Then $F$ has an invariant direction.

Proof. Essentially the same reasoning as in Theorem 2.13 (except here we use Theorem 3.7 instead of Theorem 2.6) establishes the result.

Remark 3.13. In Theorem 3.12, we can replace $B$ by any open set $U$ of $E$ with $0 \in U$ (here $E$ is any Hausdorff topological vector space) provided that $\partial U$ is a retract of $\bar{U}$, and in this case (3.13) is replaced by the following condition: $\exists \mu>0$ with $\mu F(\partial U) \cap \bar{U}=\varnothing$.

Remark 3.14. In Theorem 3.12, $F \in G A^{1}(\bar{B}, E)$ could be replaced by $F \in G A^{1}$ $(S, E)$.

Example 3.15. In Theorem 3.12, if condition $(C)$ means that the map $F: \bar{B} \rightarrow$ $K(E)$ is either (a) Kakutani, (b) acyclic, (c) O'Neill, (d) approximable, or (e) admissible (strongly) in the sense of Gorniewicz, then clearly (3.1), (3.2), (3.9), and (3.12) hold.

We also have the following result when $E$ is not necessarily infinite dimensional.

Theorem 3.16. Let $E=(E,\|\cdot\|)$ be a normed linear space, $C \subseteq E$ is a cone (i.e., closed, convex, invariant under multiplication by nonnegative real numbers and $C \cap(-C)=\{0\}), U=B_{C}, F \in G A^{1}\left(\overline{B_{C}}, C\right)$, and assume (3.1), (3.2) (with $i=1$ ), and (3.9) hold, here $B_{C}=\{x \in C:\|x\|<1\}$ and $\overline{B_{C}}=\{x \in C:\|x\| \leq 1\}$. In addition, suppose the following two conditions are satisfied:

(a) for any continuous map $r: \overline{B_{C}} \rightarrow S_{C}$, Fr satisfies condition $(C)$;

(b) $0 \notin \overline{F\left(S_{C}\right)}$,

here $S_{C}=\{x \in C:\|x\|=1\}$. Then $F$ has an invariant direction.

Proof. Since $C$ is a cone, it is well known that there exists a continuous retraction $r: \overline{B_{C}} \rightarrow S_{C}$. Let $G=F r$ and follow Theorem 2.13. 
Also, as in Section 2, if $E$ is an infinite-dimensional normed linear space, then there exists a Lipschitzian retraction $r: \bar{B} \rightarrow S$ with Lipschitz constant $k_{0}(E)$, here $B=\{x \in E:\|x\|<1\}$ and $S=\{x \in E:\|x\|=1\}$.

Theorem 3.17. Let $E=(E,\|\cdot\|)$ be an infinite-dimensional normed linear space, $C=E, U=B, F \in G A(\bar{B}, E)$, and assume (2.13), (3.1), (3.2) (with $i=3)$, (a), and (3.12) hold, here $B=\{x \in E:\|x\|<1\}$ and $S=\{x \in E:\|x\|=1\}$. In addition, suppose the following two conditions are satisfied:

(a) $F$ is countably $k$-set contractive with $0 \leq k<1 / k_{0}(E)$, here $k_{0}(E)$ is as in (2.13);

(b) there exist $\mu>0$ with $0<\mu \leq 1, \mu F(S) \cap \bar{B}=\varnothing$.

Then $F$ has an invariant direction.

Proof. Essentially the same reasoning as in Theorem 2.18 establishes the result.

\section{References}

[1] R. P. Agarwal and D. O'Regan, Homotopy and normalization properties for admissible maps, to appear.

[2] Multivalued essential maps of approximable and acyclic type, Appl. Math. Lett. 13 (2000), no. 1, 7-11.

[3] Continuation theorems for countably condensing maps, Nonlinear Funct. Anal. Appl. 6 (2001), no. 1, 1-18.

[4] Homotopy and Leray-Schauder principles for multimaps, Nonlinear Analysis Forum 7 (2002), 103-111.

[5] R. P. Agarwal, D. O'Regan, and S. Park, Fixed point theory for multimaps in extension type spaces, J. Korean Math. Soc. 39 (2002), no. 4, 579-591.

[6] H. Ben-El-Mechaiekh and P. Deguire, Approachability and fixed points for nonconvex set-valued maps, J. Math. Anal. Appl. 170 (1992), no. 2, 477-500.

[7] Y. Benyamini and Y. Sternfeld, Spheres in infinite-dimensional normed spaces are Lipschitz contractible, Proc. Amer. Math. Soc. 88 (1983), no. 3, 439-445.

[8] M. Furi and M. Martelli, A degree for a class of acyclic-valued vector fields in Banach spaces, Ann. Scuola Norm. Sup. Pisa Cl. Sci. (4) 1 (1974), 301-310.

[9] K. Goebel and W. A. Kirk, Topics in Metric Fixed Point Theory, Cambridge Studies in Advanced Mathematics, vol. 28, Cambridge University Press, Cambridge, 1990.

[10] L. Gorniewicz and M. Slosarski, Topological essentiality and differential inclusions, Bull. Austral. Math. Soc. 45 (1992), no. 2, 177-193.

[11] D. O'Regan, Furi-Pera type theorems for the $U_{c}^{\kappa}$-admissible maps of Park, to appear in Math. Proc. Royal Irish Academy.

[12] - Fixed point theorems for the $\mathscr{B}^{k}$-admissible maps of Park, Appl. Anal. 79 (2001), no. 1-2, 173-185.

[13] A Anified fixed point theory for countably P-concentrative multimaps, Appl. Anal. 81 (2002), no. 3, 565-574.

[14] S. Park, Generalized Leray-Schauder principles for condensing admissible multifunctions, Ann. Mat. Pura Appl. (4) 172 (1997), 65-85.

[15] _ A Anified fixed point theory of multimaps on topological vector spaces, J. Korean Math. Soc. 35 (1998), no. 4, 803-829. 


\section{Birkhoff-Kellogg theorems}

[16] S. Park and K. S. Jeong, The Leray-Schauder principles for condensing approximable and other multimaps, Nonlinear Anal. Forum 4 (1999), 157-173.

[17] S. Reich, A remark on set-valued mappings that satisfy the Leray-Schauder condition, Atti Accad. Naz. Lincei Rend. Cl. Sci. Fis. Mat. Natur. (8) 61 (1976), no. 3-4, 193194.

Ravi P. Agarwal: Department of Mathematical Sciences, Florida Institute of Technology, Melbourne, FL 32901-6975, USA

E-mail address: agarwal@fit.edu

Donal O’Regan: Department of Mathematics, National University of Ireland, Galway, Ireland

E-mail address: donal.oregan@nuigalway.ie 


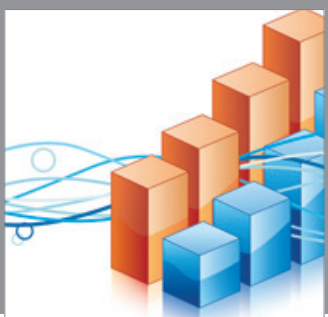

Advances in

Operations Research

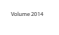

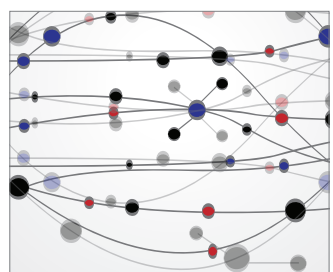

\section{The Scientific} World Journal
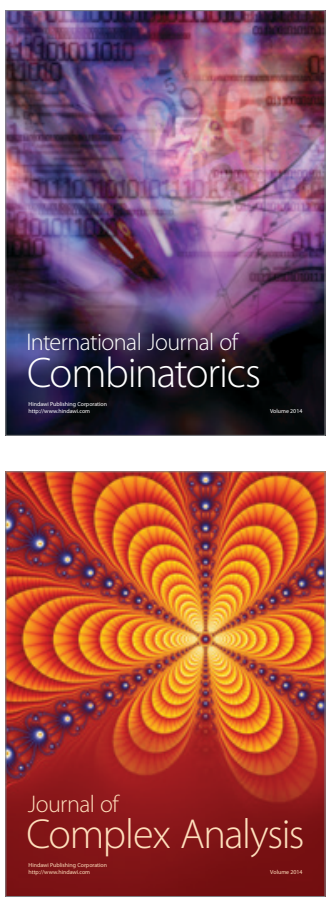

International Journal of

Mathematics and

Mathematical

Sciences
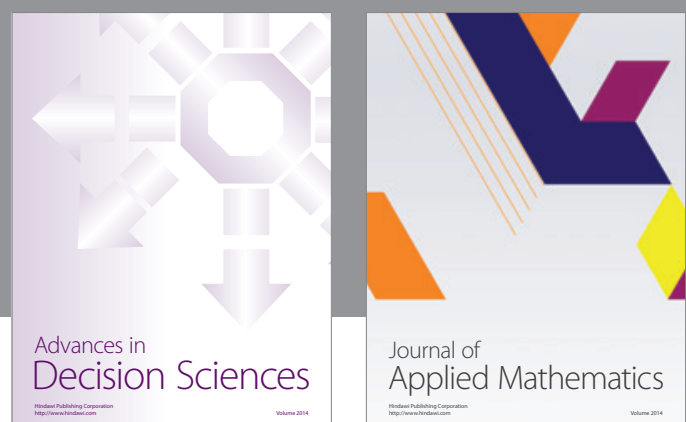

Journal of

Applied Mathematics
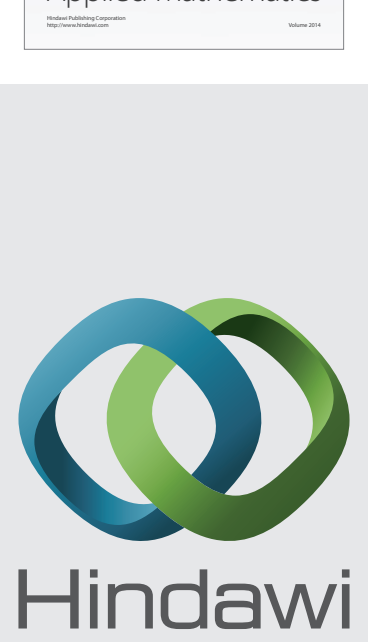

Submit your manuscripts at http://www.hindawi.com
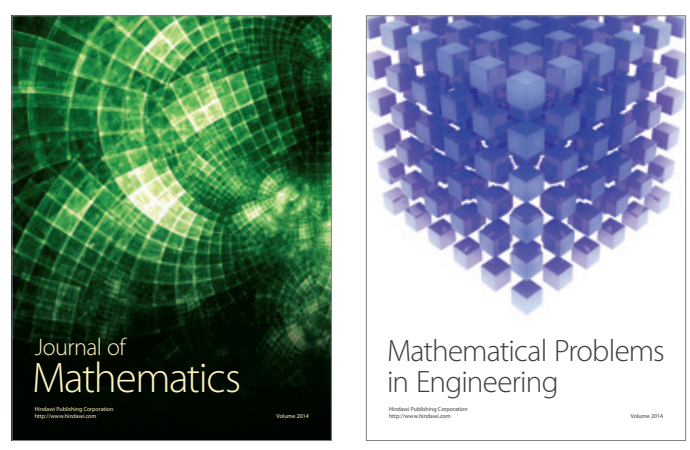

Mathematical Problems in Engineering
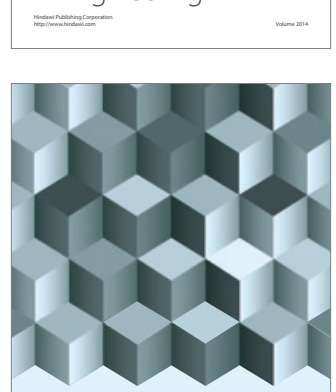

Journal of

Function Spaces
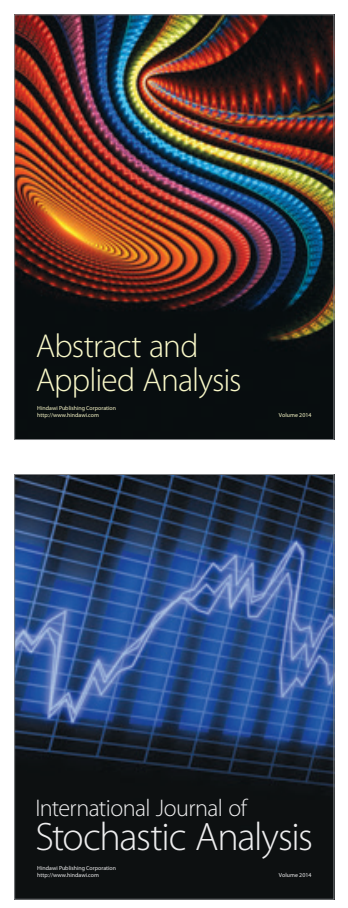

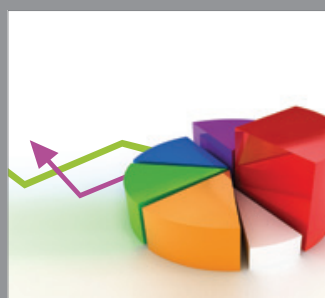

ournal of

Probability and Statistics

Promensencen
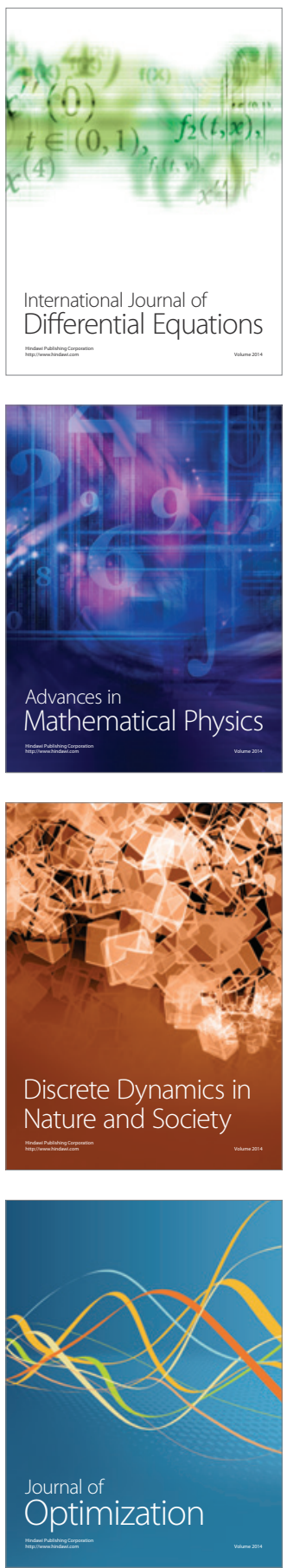